Supporting Information

\title{
Stabilization of Fibronectin by Random Copolymer Brushes Inhibits Macrophage Activation
}

\author{
David Faulón Marruecos ${ }^{1}$, Leila S. Saleh ${ }^{1}$, Hye Hyun Kim ${ }^{1}$, Stephanie J. Bryant ${ }^{1,2,3}$, Daniel K. \\ Schwartz $^{1 *}$, Joel L. Kaar ${ }^{*}$ \\ ${ }^{1}$ Department of Chemical and Biological Engineering, University of Colorado, Boulder, CO \\ 80309, USA \\ ${ }^{2}$ BioFrontiers Institute, University of Colorado, Boulder, CO 80309, USA \\ ${ }^{3}$ Material Science and Engineering Program, University of Colorado, Boulder, CO 80309, USA \\ *Corresponding Authors: Email: joel.kaar@colorado.edu and daniel.schwartz@,colorado.edu
}

This PDF file includes:

Experimental Details

Tables S1 and S2

Figs. S1 to S8

References 


\section{EXPERIMENTAL DETAILS}

\section{FN Production for SM Analysis}

For SM studies, the same FRET-labeled FN construct as used in previous work was used here $^{1,2}$. Briefly, the construct contained the FN type III 8-10 domains and was expressed containing the non-native amino acid $p$-azidophenylalanine and a unique cysteine in BL21 (DE3) Escherichia coli. Incorporation of $p$-azidophenylalanine was enabled via co-expression with pDule2 pCNF RS in minimal medial supplemented with the non-native amino acid $^{3,4}$. Following expression, the mutant protein was purified via Ni-IDA affinity chromatography and subsequently labeled with dibenzocyclooctyne-Alexa Fluor 555 and maleimide-Alexa Fluor 594 (Life Technologies). Based on the molar extinction coefficients for the dyes and for the FN construct, the labeling efficiency for Alexa Fluor 555 and 647 was estimated to be $70 \%$ and $40 \%$, respectively.

\section{Preparation and Characterization of Polymer Brush Surfaces}

Initially, polymer brush surfaces for SM analysis as well as in vitro macrophage activation assays were prepared by generating a self-assembled monolayer of the ATRP initiator ((chloromethyl)phenylethyl)trichlorosilane (CMPS, Gelest) on two-inch fused silica wafers (Mark Optics). Prior to formation of the self-assembled monolayer, the wafers were cleaned with piranha for $2 \mathrm{~h}$ and subsequently treated with ozone plasma for $30 \mathrm{~min}$. The treated surfaces were then reacted with a $0.5 \mathrm{mM}$ initiator solution in pure toluene for $1 \mathrm{~h}$ before finally rinsing off the surfaces with toluene several times and drying the surfaces under nitrogen ${ }^{5}$.

Polymer brushes were grown from the initiator monolayer via surface-initiated ATRP using a $0: 1,1: 3,1: 1,3: 1,1: 0$ molar feed ratio of [2-(methacryloyloxy)ethyl]dimethyl-(3sulfopropyl)ammonium hydroxide (SBMA, Sigma Aldrich) to poly(ethylene glycol) methacrylate (OEGMA, $M_{\mathrm{n}} 360 \mathrm{Da}$, Sigma Aldrich). The monomers were dissolved in a solution (15 mL) with 
4 parts water and 1 part methanol by volume at a total monomer concentration of $500 \mathrm{mM}$. After purging the monomer solution with nitrogen for at least $30 \mathrm{~min}$, the solution was transferred to a Schlenk flask containing copper bromide (23 mg) and 2,2'-bipyridyl (50 mg), which was dried in an oven and deoxygenated via three vacuum-nitrogen freeze-thaw-pump cycles. The reaction solution was vortexed until homogenization and subsequently transferred to a customized reactor, which was under positive nitrogen pressure, containing the initiator-modified wafers. After $2 \mathrm{~h}$ at $60{ }^{\circ} \mathrm{C}$, the polymerization reaction was stopped by exposing the reaction solution to atmospheric oxygen.

Following several washes with warm water and methanol and drying under nitrogen, the dry thickness of the brushes was measured using a VASE-VB-250 J.A. Woollam variable angle ellipsometer. For ellipsometry measurements, spectra of the brush surfaces were obtained by scanning the surfaces between $500 \mathrm{~nm}$ and $900 \mathrm{~nm}$ with an incident angle between $60^{\circ}$ and $80^{\circ}$ (in increments of $5^{\circ}$ ). The spectra were fit to a three-layer planar model while using values for the refractive index of air, OEGMA, SBMA, silicone dioxide, and silicon of 1.003, 1.450, 1.450, 1.457, and 3.881, respectively. Note, for all surface characterization, the brush surfaces were prepared on silicon wafers instead of fused silica wafers, although the same procedure for growing the brushes was used. Additionally, the sessile-drop water contact angle for each surface was measured using a custom-built goniometer and a droplet size of $1 \mu \mathrm{L}$ at three independent locations. Finally, using a Nicolet 6700 FTIR (Thermo Fisher), infrared spectra (1000 scans) of the brush surfaces and initiator-coated surfaces, which was used as the background, were collected with $1 \mathrm{~cm}^{-1}$ resolution. 


\section{Collection and Processing of SM-FRET Data}

SM-FRET experiments of FRET-labeled FN in contact with polymer brush-coated glass were performed as explained in detail elsewhere using a TIRFM microscope ${ }^{1,6}$. In summary, a

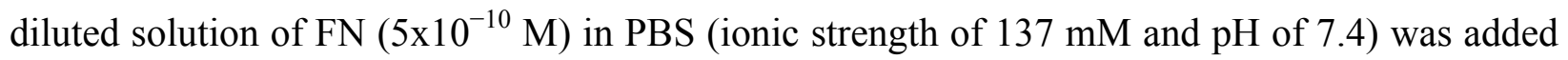
to a flow cell containing the polymer brush-coated fused silica surface, maintained at $25.0 \pm 0.1$ ${ }^{\circ} \mathrm{C}$. The flow cell was placed into a custom-built illumination system, where a cylindrical prism is placed on top of the flow cell to allow for total internal reflection of an incident $532 \mathrm{~nm}$ diodepumped solid-state laser (Samba, Cobolt) at the solid-liquid interface. The microscope used was a Nikon TE-2000 with 60x plan Apo water immersion objective and $1.5 \mathrm{x}$ post-objective magnification lens. Fluorescence emission from the donor and acceptor dyes of the labeled protein molecules at the near-surface environment was split into two different channels. For spectral separation, an Optosplit III beam splitter (Cairn Research) containing a $610 \mathrm{~nm}$ dichroic mirror (T610LPXR, Chroma) was used. For further spectral selection and avoidance of bleed-through, the emission spectra from the donor and the acceptor dyes were filtered with a 585/40 and a $685 / 40$ bandpass filter, respectively (90\% transmission, Semrock). Fluorescence intensity from each channel (i.e. donor and acceptor) was collected separately by an Andor iXon-888 EMCCD camera (model No. DU897; Andor Technology, Belfast, UK) operating at $-90^{\circ} \mathrm{C}$ with a frame acquisition time of $100 \mathrm{~ms}$.

Objects collected in each channel were identified and connected into trajectories as explained in detail elsewhere ${ }^{7,8}$. Adsorption rate constants were calculated as the total number of adsorption events on each surface divided by the duration of each movie, the area of the field of view and protein concentration in solution. In order to include a molecule in the calculation, it had 
to appear in both the donor and acceptor channel in at least one frame throughout the duration of its trajectory and reside on the surface for at least 4 frames.

Rates of folding, unfolding and desorption were calculated as described previously in detail ${ }^{2,8,9}$. Briefly, complementary cumulative dwell time and surface residence time distributions of the molecules were constructed for each surface. Specifically, dwell times referred to the time that an individual molecule spends on the surface in a given conformation before transitioning into another (i.e. undergoing a conformational change), while surface residence times referred to the time that an individual molecule spends on the surface in a given conformation before desorbing (i.e. without undergoing any conformational change). A threshold value of $d=1.1$, which corresponded to the local minimum in the d-value distributions represented in Figure 3, was used to classify molecules as folded (if $d<1.1$ ) or unfolded (if $d>1.1$ ). As did before, objects with residence times of less than 4 frames were not included into the analyses to avoid random noise. The accumulated distributions were subsequently fit to an exponential mixture model $F(t)=$ $A^{-1} \sum_{k=1}^{N} x_{k} e^{-t / \tau_{k}}$, where $F(t)$ is the probability that an object has a residence time $\geq t, x_{k}$ is the relative fraction of all the objects that follows pathway $k$, and $\tau_{k}$ represents the characteristic dwell or surface residence time of that pathway ${ }^{10}$.

\section{Super-resolution Mapping of Polymer Brushes with NBD-X}

A dilute solution of the environmentally-sensitive probe NBD-X $\left(10^{-7} \mathrm{M}\right)$ in DDI water was introduced into a flow cell (maintained at $25.0 \pm 0.1^{\circ} \mathrm{C}$ ) containing the polymer brush-coated fused silica surface. The sample was excited using a $491 \mathrm{~nm}$ Cobolt Samba $50 \mathrm{~mW}$ diode-pumped solid-state laser via total internal reflection. For imaging, the same prism-based illumination system described in the previous section was used. To collect the images, an Optosplit III (Cairn 
Research) image splitter containing a dichroic mirror with nominal separation wavelength of 540 nm (model T540lpxr, Chroma) was employed. To further delimit the fluorescence emitted by the probe in hydrophobic environments, an additional bandpass filter (Semrock) centered at 528 with a $90 \%$ transmission width of $28 \mathrm{~nm}$ was utilized. Images were obtained with an acquisition time of 100 ms using the same Andor iXon-888 EMCCD camera described above.

The collected images were analyzed using the motion blur point accumulation for imaging in nanoscale topography (mbPAINT) method, as described previously in detail ${ }^{11-13}$. Briefly, from the accumulation of $\sim 80,000$ adsorption events of the NBD-X molecules throughout each surface, super-resolution spatial maps of hydrophobic spots were constructed. Specifically, each adsorption event represented the activation of the probe in a low dielectric (i.e. hydrophobic) environment within the polymer brush. The adsorption maps were generated by binning the number of adsorption events onto a pseudoimage with $15 \mathrm{~nm}$ pixels for the same number of randomly selected trajectories at each condition. A Gaussian blur was applied to this pseudoimage with a radius of $100 \mathrm{~nm}$ to account for positional uncertainty.

\section{Macrophage Culture and Assays for Adhesion and Activation}

The activation of macrophages on the polymer brush surfaces was measured using early passage murine macrophage cells from the cell line RAW 264.7 (ATCC, TIB-71). The cells were expanded in DMEM (Gibco) supplemented with 10\% FBS (Atlanta Biologicals), $50 \mathrm{U} / \mathrm{mL}$ penicillin, $50 \mu \mathrm{g} / \mathrm{mL}$ streptomycin (1\% P/S, Corning), and 0.5\% Fungizone (Corning). Following expansion, the cells were cultured on tissue culture polystyrene (TCPS) to $\sim 85 \%$ confluency and then collected using a cell scraper prior to culture on the modified surfaces described above. Prior to culture on the modified brush surface, the surfaces were soaked in $150 \mu \mathrm{g} / \mathrm{mL}$ full-length human 
fibronectin (FN, EMD Millipore) for $2 \mathrm{~h}$ at room temperature. Macrophages were then plated on the FN-coated surfaces at 2650 cells $/ \mathrm{mm}^{2}$ in serum-free medium containing DMEM, 1\% $\mathrm{ITS}^{\mathrm{TM}}+$ Premix universal culture supplement (Corning), 1\% MEM non-essential amino acids (Corning), $1 \% \mathrm{P} / \mathrm{S}$, and $0.5 \%$ Fungizone.

To characterize macrophage adhesion and activation, media samples were collected after culturing the cells on the brush surface for $4 \mathrm{~h}$ and then after $24 \mathrm{~h}$ of culture the surfaces were washed and the cells fixed in 4\% paraformaldehyde. Immunocytochemical analysis was performed to visualize cell attachment and morphology at $24 \mathrm{~h}$. Samples were permeabilized and blocked, then incubated with rat anti-mouse F4/80 antibody (1:50, BioLegend, BM8), a marker of macrophages, in blocking solution overnight at $4^{\circ} \mathrm{C}$. After blocking, the samples were treated with a secondary AlexaFluor 488 donkey anti-rat antibody (1:200, Invitrogen) for $2 \mathrm{~h}$ at room temperature. The samples were also counterstained for F-actin using AlexaFluor 546-Phalloidin (1:30, Invitrogen) for $30 \mathrm{~min}$ at room temperature and then counterstained with DAPI for nuclei. Images of the samples were obtained via spinning disc confocal microscopy using a Nikon Ti-E microscope located in the BioFrontiers Advanced Light Microscopy Core. Macrophage activation was assessed for the presence of tumor necrosis factor- $\alpha$ (TNF- $\alpha$ ) and interleukin-6 (IL-6) in the media after $4 \mathrm{~h}$ by enzyme-linked immunosorbent assay using commercial kits (R\&D Systems DuoSet) based on a sandwich format and detection via the activity of horseradish peroxidase. Data are presented as the mean of three replicates with standard deviation as error bars. For image analysis, data are presented as the mean of three replicates with four images per replicate. Statistical analysis was performed using a one-way analysis of variance (ANOVA) with surface zwitterionic content as the factor followed by Tukey's post hoc analysis with alpha $=0.05$. 


\section{SUPPORTING TABLES}

Table S1. Quantitative analysis of FTIR spectra of poly(OEGMA-co-SBMA) brushes. Peaks corresponding to the backbone of the brush $\left(\mathrm{C}=\mathrm{O}\right.$ stretching, centered at $\left.1730 \mathrm{~cm}^{-1}\right)$, OEGMA monomers $\left(\mathrm{C}-\mathrm{O}\right.$ stretching, centered around $\left.1083 \mathrm{~cm}^{-1}\right)$, and SBMA monomers $\left(\mathrm{SO}_{3}{ }^{-}\right.$asymmetric stretching, centered at $1193 \mathrm{~cm}^{-1}$, as well as $\mathrm{SO}_{3}^{-}$symmetric stretching, centered at $1041 \mathrm{~cm}^{-1}$ ) were integrated and displayed on the table as peak areas. The peak area of each monomer was then divided by the peak area of the backbone in order to normalize by the "amount" of brush present in the sample and allow for comparison between monomers. Using as a reference (i.e. calibration) the normalized values corresponding to pure OEGMA and pure SBMA brushes, the percentages of each monomer in the mixed brushes were calculated. Representative spectra are shown in Figure S1B.

\begin{tabular}{|c|c|c|c|c|c|c|c|c|c|c|}
\hline \multirow[b]{3}{*}{$\begin{array}{l}\text { Zwitterio } \\
\quad \text { nic } \\
\text { content in } \\
\text { feed }(\%)\end{array}$} & \multirow[b]{3}{*}{$\begin{array}{c}\text { Peak } \\
\text { area } \\
(C=0 \\
\text { stretchi } \\
\text { ng) }\end{array}$} & \multirow[b]{3}{*}{$\begin{array}{c}\text { Peak } \\
\text { area } \\
\text { (C-O } \\
\text { stretchi } \\
\text { ng) }\end{array}$} & \multirow[b]{3}{*}{$\begin{array}{l}\text { Peak area } \\
\quad\left(\mathrm{SO}_{3}^{-}\right. \\
\text {asymmetric } \\
\text { stretching) }\end{array}$} & \multirow[b]{3}{*}{$\begin{array}{l}\text { Peak area } \\
\quad\left(\mathrm{SO}_{3}^{-}\right. \\
\text {symmetric } \\
\text { stretching) }\end{array}$} & \multirow{2}{*}{\multicolumn{3}{|c|}{$\begin{array}{c}\text { Normalized by backbone } \\
\text { peak }(C=O \text { stretching) }\end{array}$}} & & & \\
\hline & & & & & & & & \multicolumn{3}{|c|}{ Brush composition (\%) } \\
\hline & & & & & $\begin{array}{l}\text { C-O } \\
\text { stretch } \\
\text { ing }\end{array}$ & $\begin{array}{c}\mathrm{SO}_{3}^{-} \\
\text {asym } \\
\text { metri } \\
\text { c } \\
\text { stretc } \\
\text { hing } \\
\end{array}$ & $\begin{array}{c}\mathrm{SO}_{3}^{-} \\
\text {symmet } \\
\text { ric } \\
\text { stretchi } \\
\text { ng }\end{array}$ & $\begin{array}{c}\text { OEG } \\
\text { MA }\end{array}$ & $\begin{array}{c}\text { SBMA } \\
\text { (from } \\
\text { asym. } \\
\text { stretch. } \\
\text { peak) }\end{array}$ & $\begin{array}{c}\text { SBMA } \\
\text { (from } \\
\text { sym. } \\
\text { stretch. } \\
\text { peak) }\end{array}$ \\
\hline $\mathbf{0}$ & 0.430 & 0.267 & 0.000 & 0.000 & 0.621 & 0.000 & 0.000 & 100 & 0 & 0 \\
\hline 25 & 0.433 & 0.196 & 0.137 & 0.039 & 0.453 & 0.317 & 0.090 & 73 & 23 & 26 \\
\hline 50 & 0.436 & 0.142 & 0.284 & 0.074 & 0.325 & 0.653 & 0.169 & 52 & 48 & 48 \\
\hline 75 & 0.438 & 0.059 & 0.443 & 0.110 & 0.135 & 1.011 & 0.252 & 22 & 74 & 71 \\
\hline 100 & 0.436 & 0.000 & 0.593 & 0.154 & 0.000 & 1.360 & 0.353 & 0 & 100 & 100 \\
\hline
\end{tabular}


Table S2. Fitting parameters for integrated state dwell time distributions on poly(OEGMA-coSBMA) brushes for folded and unfolded FN. The parameters were determined by fitting the complementary cumulative dwell time distributions for folded and unfolded FN to an exponential mixture model with multiple populations $(k)$. Each population was characterized with a relative fraction $\left(x_{k}\right)$ and characteristic time $\left(\tau_{k}\right)$. Additionally, $\langle\tau\rangle$ and $k_{\text {fold/unf }}\left(\langle\tau\rangle^{-1}\right)$, represent the mean state dwell time and the mean characteristic rate constant for folding or unfolding, respectively. These mean parameters were determined from the weighted average of the individual population fractions times the population state dwell times. The numbers in parentheses correspond to the uncertainty in the least significant digit, and represent the standard error of the weighted fit values.

\begin{tabular}{|c|c|c|c|c|}
\hline $\begin{array}{c}\text { Surface, Dwell State } \\
\text { (Conformational change) }\end{array}$ & $\langle\boldsymbol{\tau}\rangle(\mathbf{s})$ & $k_{\text {fold } / \text { unf }}\left(\mathrm{s}^{-1}\right)$ & $x_{\mathrm{k}}$ & $\tau_{\mathrm{k}}(\mathbf{s})$ \\
\hline \multirow{3}{*}{$\begin{array}{c}\left.\text { p(SBMA } \text { SM-co-OEGMA }_{100}\right) \\
\text { Unfolded (Folding) }\end{array}$} & \multirow{3}{*}{$23.2(4)$} & \multirow{3}{*}{$0.0431(7)$} & $0.481(3)$ & $0.159(5)$ \\
\hline & & & $0.162(2)$ & $2.05(5)$ \\
\hline & & & $0.357(4)$ & $63.9(7)$ \\
\hline \multirow{3}{*}{$\begin{array}{c}\left.\text { p(SBMA } \text { SE-co-OEGMA }_{75}\right) \\
\text { Unfolded (Folding) }\end{array}$} & \multirow{3}{*}{$19.3(4)$} & \multirow{3}{*}{$0.052(1)$} & $0.616(3)$ & $0.152(3)$ \\
\hline & & & $0.128(2)$ & $2.20(5)$ \\
\hline & & & $0.256(4)$ & $74(1)$ \\
\hline \multirow{3}{*}{$\begin{array}{c}\left.\text { p(SBMA } \text { So-co-OEGMA }_{50}\right) \\
\text { Unfolded (Folding) }\end{array}$} & \multirow{3}{*}{$12.4(4)$} & \multirow{3}{*}{$0.081(3)$} & $0.682(3)$ & $0.166(5)$ \\
\hline & & & $0.150(3)$ & $1.95(6)$ \\
\hline & & & $0.169(4)$ & $71(2)$ \\
\hline \multirow{3}{*}{$\begin{array}{c}\text { p( } \text { SBMA }_{75} \text {-co-OEGMA } \\
\text { Unfolded (Folding) }\end{array}$} & \multirow{3}{*}{$6.1(2)$} & \multirow{3}{*}{$0.164(5)$} & $0.689(3)$ & $0.160(4)$ \\
\hline & & & $0.167(3)$ & $1.70(4)$ \\
\hline & & & $0.144(4)$ & $39.5(5)$ \\
\hline \multirow{3}{*}{$\begin{array}{c}\left.\text { p(SBMA } \text { SBo-co-OEGMA }_{10}\right) \\
\text { Unfolded (Folding) }\end{array}$} & \multirow{3}{*}{$11.6(2)$} & \multirow{3}{*}{$0.086(2)$} & $0.480(4)$ & $0.152(5)$ \\
\hline & & & $0.200(2)$ & $1.54(3)$ \\
\hline & & & $0.319(5)$ & $35.0(2)$ \\
\hline \multirow{3}{*}{$\begin{array}{l}\left.\text { p(SBMA } \text { SB-co-OEGMA }_{100}\right) \\
\text { Folded (Unfolding) }\end{array}$} & \multirow{3}{*}{$0.31(3)$} & \multirow{3}{*}{$3.2(3)$} & $0.848(7)$ & $0.094(6)$ \\
\hline & & & $0.104(6)$ & $0.63(4)$ \\
\hline & & & $0.048(9)$ & $3.36(3)$ \\
\hline \multirow{3}{*}{$\begin{array}{l}\left.\text { p(SBMA } \text { (S5-co-OEGMA }_{75}\right) \\
\text { Folded (Unfolding) }\end{array}$} & \multirow{3}{*}{$0.73(6)$} & \multirow{3}{*}{$1.4(1)$} & $0.823(4)$ & $0.228(7)$ \\
\hline & & & $0.156(3)$ & 1.91(4) \\
\hline & & & $0.021(5)$ & $11.4(2)$ \\
\hline \multirow{3}{*}{$\begin{array}{l}\left.\text { p(SBMA } \text { SO-co-OEGMA }_{50}\right) \\
\text { Folded (Unfolding) }\end{array}$} & \multirow{3}{*}{$1.2(1)$} & \multirow{3}{*}{$0.83(7)$} & $0.739(9)$ & $0.31(2)$ \\
\hline & & & $0.224(7)$ & $2.37(9)$ \\
\hline & & & $0.04(1)$ & $9.7(4)$ \\
\hline \multirow{3}{*}{$\begin{array}{l}\left.\text { p(SBMA } \text { SF-co-OEGMA }_{25}\right) \\
\text { Folded (Unfolding) }\end{array}$} & \multirow{3}{*}{$2.1(2)$} & \multirow{3}{*}{$0.48(5)$} & $0.729(4)$ & $0.230(8)$ \\
\hline & & & $0.228(3)$ & $3.00(7)$ \\
\hline & & & $0.043(5)$ & $30(2)$ \\
\hline \multirow{3}{*}{$\begin{array}{l}\left.\text { p(SBMA } \text { SBo-co-OEGMA }_{10}\right) \\
\text { Folded (Unfolding) }\end{array}$} & \multirow{3}{*}{$0.18(1)$} & \multirow{3}{*}{$5.6(3)$} & $0.869(5)$ & $0.078(3)$ \\
\hline & & & $0.102(4)$ & $0.41(1)$ \\
\hline & & & $0.029(6)$ & $2.26(2)$ \\
\hline
\end{tabular}


Table S3. Fitting parameters for integrated surface residence time distributions on poly(OEGMAco-SBMA) brushes for folded and unfolded FN. The reported parameters were determined by fitting the complementary cumulative surface residence time distributions for folded and unfolded FN to an exponential mixture model with multiple populations $(k)$. Each population was characterized with a relative fraction $\left(x_{k}\right)$ and characteristic time $\left(\tau_{k}\right)$. Additionally, $\langle\tau\rangle$ and $k_{\text {fold/unf }}$ $\left(\langle\tau\rangle^{-1}\right)$, represent the mean surface residence time and the mean characteristic desorption rate constant, respectively. These mean parameters were determined from the weighted average of the individual population fractions times the population residence times. The numbers in parentheses correspond to the uncertainty in the least significant digit, and represent the standard error of the weighted fit values.

\begin{tabular}{|c|c|c|c|c|}
\hline Surface (State) & $\langle\boldsymbol{\tau}\rangle(\mathbf{s})$ & $k_{\text {des }}\left(\mathrm{s}^{-1}\right)$ & $x_{k}$ & $\tau_{\mathrm{k}}(\mathbf{s})$ \\
\hline \multirow{4}{*}{$\begin{array}{c}\left.\text { p(SBMA } \text { (SO-Co-OEGMA }_{100}\right) \\
\text { (Folded) }\end{array}$} & \multirow{4}{*}{$0.22(1)$} & \multirow{4}{*}{$4.6(2)$} & $0.661(6)$ & $0.108(2)$ \\
\hline & & & $0.297(6)$ & $0.325(5)$ \\
\hline & & & $0.042(9)$ & $1.11(1)$ \\
\hline & & & $0.00045(2)$ & $8.0(3)$ \\
\hline \multirow{4}{*}{$\begin{array}{c}\left.\text { p(SBMA } \text { (SB-Co-OEGMA }_{100}\right) \\
\text { (Unfolded) }\end{array}$} & \multirow{4}{*}{$0.92(3)$} & \multirow{4}{*}{$1.09(4)$} & $0.513(8)$ & $0.174(4)$ \\
\hline & & & $0.373(7)$ & $0.62(1)$ \\
\hline & & & $0.09(1)$ & $3.06(3)$ \\
\hline & & & $0.0229(2)$ & $14.28(6)$ \\
\hline \multirow{4}{*}{$\begin{array}{c}\left.\text { p(SBMA } \text { (S5-co-OEGMA }_{75}\right) \\
\text { (Folded) }\end{array}$} & \multirow{4}{*}{$0.221(8)$} & \multirow{4}{*}{$4.5(2)$} & $0.765(4)$ & $0.111(1)$ \\
\hline & & & $0.211(3)$ & $0.437(8)$ \\
\hline & & & $0.022(5)$ & $1.53(4)$ \\
\hline & & & $0.0017(1)$ & $6.3(2)$ \\
\hline \multirow{4}{*}{$\begin{array}{c}\left.\text { p(SBMA } \text { (S-co-OEGMA }_{75}\right) \\
\text { (Unfolded) }\end{array}$} & \multirow{4}{*}{$0.492(7)$} & \multirow{4}{*}{$2.03(3)$} & $0.753(2)$ & $0.093(1)$ \\
\hline & & & $0.192(1)$ & $0.612(7)$ \\
\hline & & & $0.044(2)$ & $3.06(3)$ \\
\hline & & & $0.0106(1)$ & $16.05(8)$ \\
\hline \multirow{4}{*}{$\begin{array}{c}\left.\text { p(SBMA } \text { (S0-co-OEGMA }_{50}\right) \\
\text { (Folded) }\end{array}$} & \multirow{4}{*}{$0.39(2)$} & \multirow{4}{*}{$2.6(1)$} & $0.660(8)$ & $0.193(2)$ \\
\hline & & & $0.300(6)$ & $0.60(1)$ \\
\hline & & & $0.04(1)$ & $1.78(5)$ \\
\hline & & & $0.0025(2)$ & $6.2(2)$ \\
\hline \multirow{4}{*}{$\begin{array}{c}\left.\text { p(SBMA } \text { (S0-co-OEGMA }_{50}\right) \\
\text { (Unfolded) }\end{array}$} & \multirow{4}{*}{$0.46(2)$} & \multirow{4}{*}{$2.2(1)$} & $0.725(4)$ & $0.134(2)$ \\
\hline & & & $0.231(4)$ & $0.596(9)$ \\
\hline & & & $0.037(6)$ & $2.98(5)$ \\
\hline & & & $0.0078(1)$ & $14.2(1)$ \\
\hline \multirow{4}{*}{$\begin{array}{c}\left.\text { p(SBMA } \text { SF-co-OEGMA }_{75}\right) \\
\text { (Folded) }\end{array}$} & \multirow{4}{*}{$0.50(2)$} & \multirow{4}{*}{$2.00(8)$} & $0.692(4)$ & $0.214(2)$ \\
\hline & & & $0.278(4)$ & $0.82(1)$ \\
\hline & & & $0.030(6)$ & $3.48(7)$ \\
\hline & & & $0.0010(2)$ & $14(1)$ \\
\hline \multirow{4}{*}{$\begin{array}{c}\left.\text { p(SBMA } \text { (S5-co-OEGMA }_{25}\right) \\
\text { (Unfolded) }\end{array}$} & \multirow{4}{*}{$0.256(9)$} & \multirow{4}{*}{$3.9(1)$} & $0.831(2)$ & $0.088(1)$ \\
\hline & & & $0.145(2)$ & $0.496(6)$ \\
\hline & & & $0.020(3)$ & $2.75(4)$ \\
\hline & & & $0.00370(6)$ & $15.0(1)$ \\
\hline \multirow{3}{*}{$\begin{array}{c}\text { p(SBMA } \text { A }_{100}-\text {-co-OEGMA } \\
\text { (Folded) }\end{array}$} & \multirow{3}{*}{$0.229(5)$} & \multirow{3}{*}{$4.4(1)$} & $0.718(8)$ & $0.131(3)$ \\
\hline & & & $0.266(8)$ & $0.427(8)$ \\
\hline & & & $0.015(1)$ & $1.39(3)$ \\
\hline
\end{tabular}




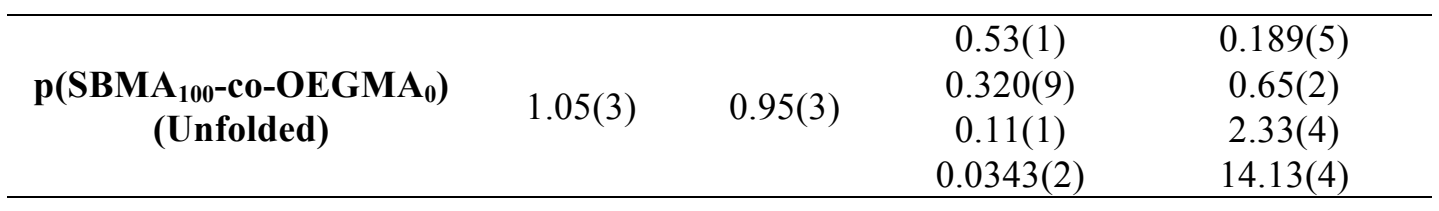




\section{SUPPORTING FIGURES}
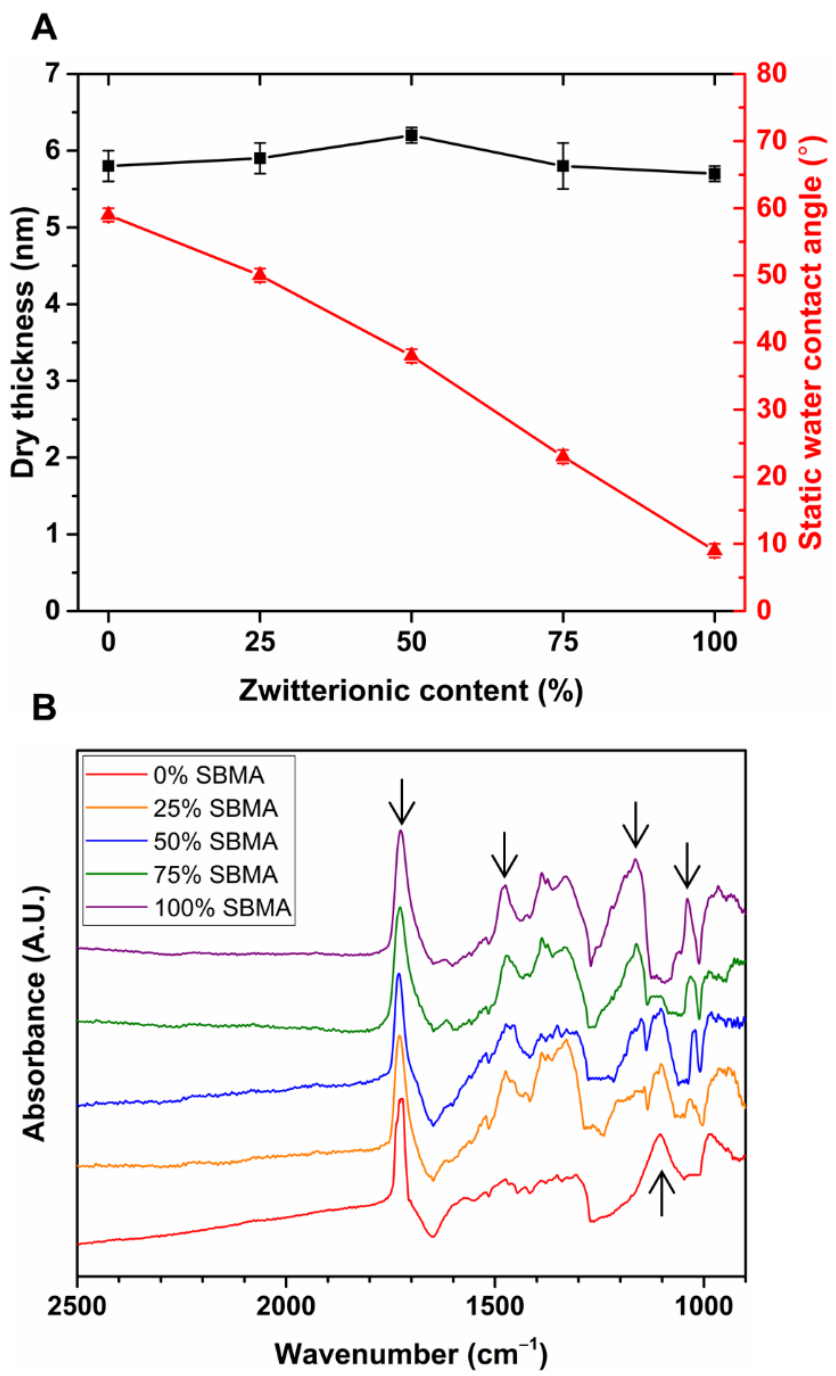

Figure S1. A) Dry thickness of poly(OEGMA-co-SBMA) brushes (black squares), as measured with ellipsometry. Static water contact angle of synthesized polymer brushes (red triangles), as measured via sessile drop technique using Milli-Q water. Contact angle measurements were also tested using PBS, but no significant differences were observed. All measurements were made at $25^{\circ} \mathrm{C}$. B) Fingerprint region of ATR-FTIR spectra for the brushes. Characteristic adsorption bands are indicated with arrows: $1730 \mathrm{~cm}^{-1}(\mathrm{C}=\mathrm{O}$ stretching $), 1485 \mathrm{~cm}^{-1}(\mathrm{C}-\mathrm{N}$ stretching $), 1193 \mathrm{~cm}^{-1}$ ( $\mathrm{SO}_{3}{ }^{-}$asymmetric stretching), $1041 \mathrm{~cm}^{-1}\left(\mathrm{SO}_{3}{ }^{-}\right.$symmetric stretching), and $1063-1103 \mathrm{~cm}^{-1}(\mathrm{C}-\mathrm{O}$ stretching). 
A
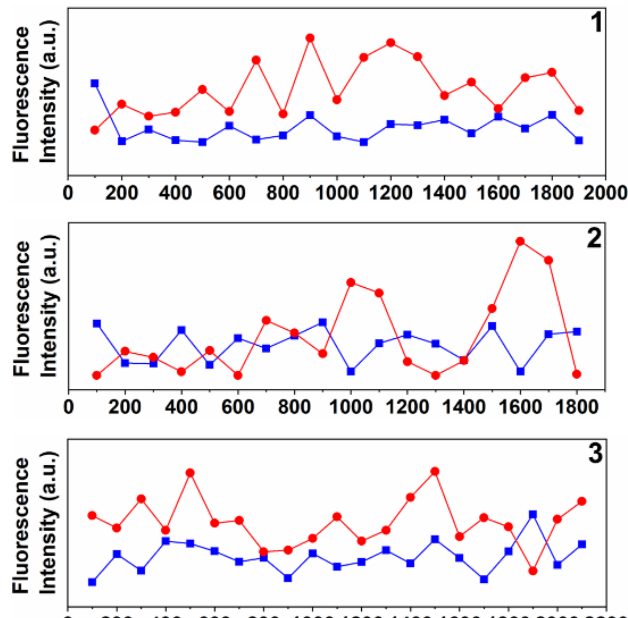

$0 \quad 200 \quad 400 \quad 600 \quad 800 \quad 1000120014001600180020002200$
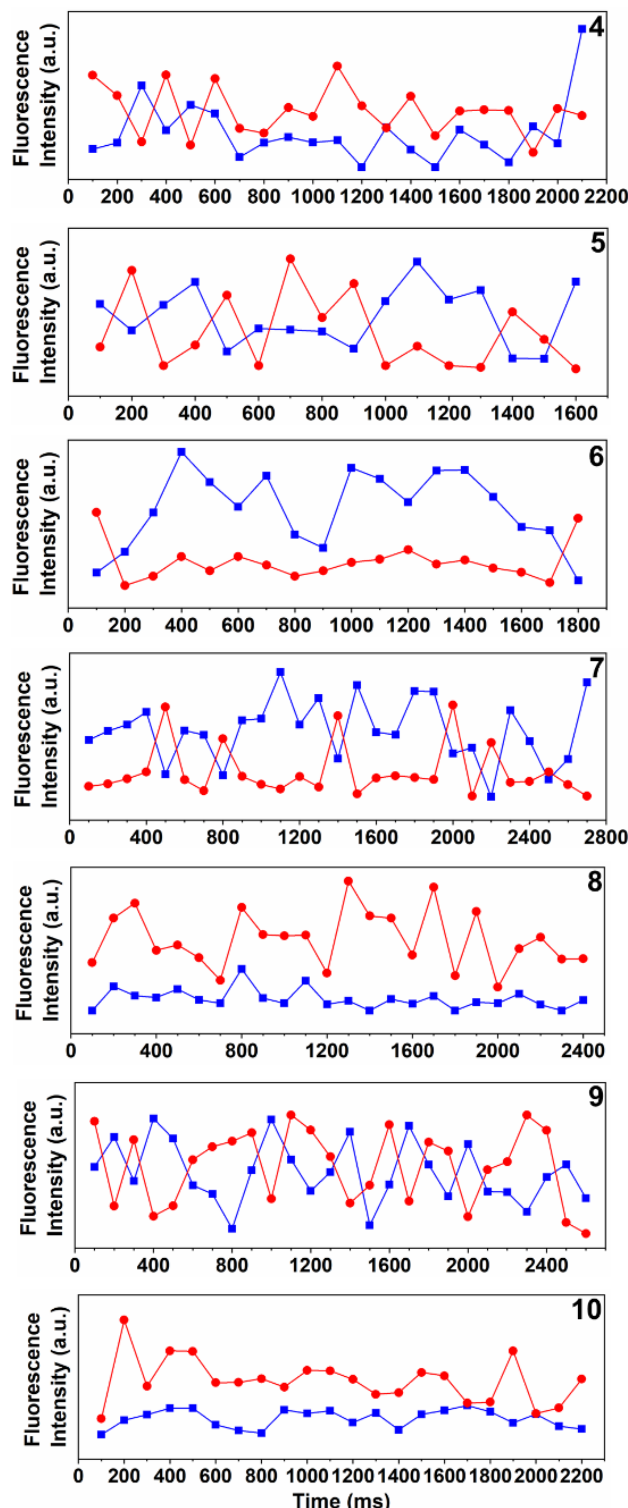

$$
\because-F_{D} \longrightarrow F_{A}
$$
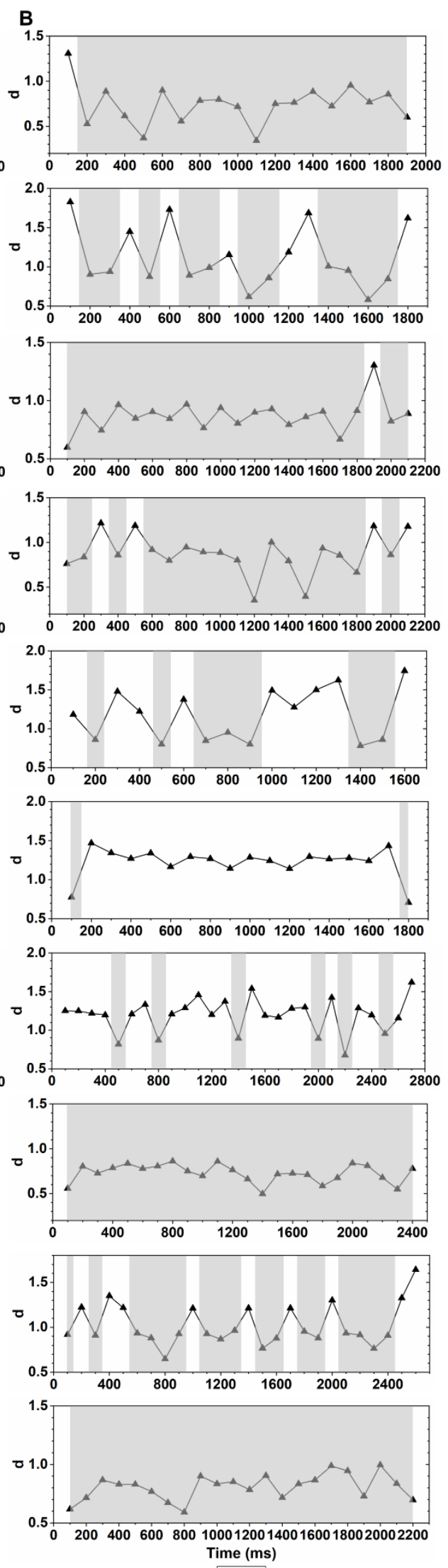

$-\mathbf{d}$ 
Figure S2. Additional randomly selected SM-FRET trajectories of individual FNIII 8-10 molecules on poly(OEGMA-co-SBMA) brush surfaces with varying OEGMA-to-SBMA ratios, showing representative raw data from SM experiments. (A) Time-dependent emission intensity traces of the donor (Alexa Fluor 555) and acceptor (Alexa Fluor 647) fluorophores for individual molecules of FRET-labeled FNIII 8-10. (B) The corresponding traces of the relative fluorophoreto-fluorophore distance, $d$, which was calculated from the raw donor and acceptor intensities and used to determine the instantaneous folding state. The shaded areas represent time intervals within a given trajectory where FNIII 8-10 was folded as determined from the value of $d$.

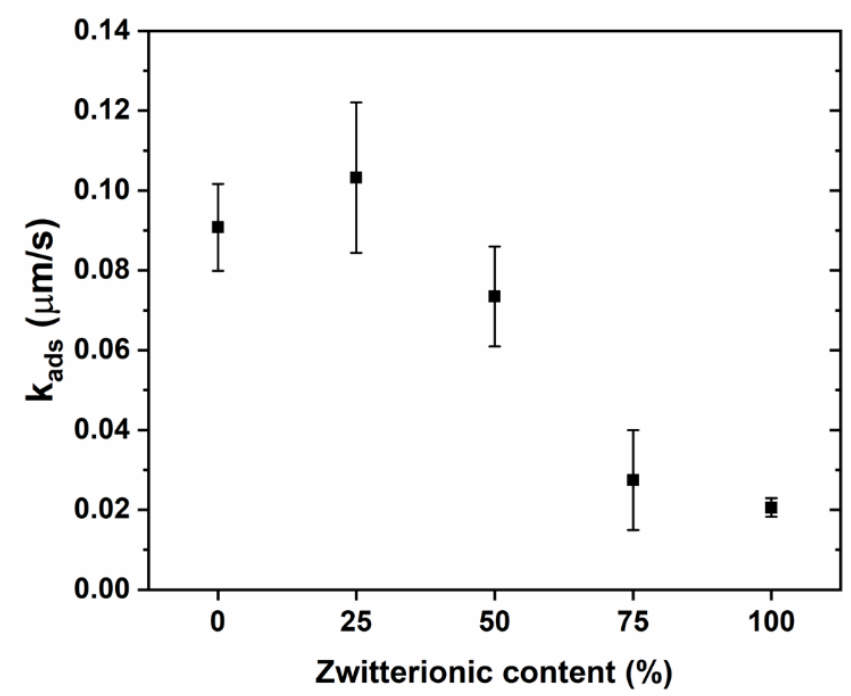

Figure S3. Adsorption rate constants of FN at room temperature as a function of polymer brush composition. Error bars represent the standard deviation across 20 data sets for each experiment. Adsorption rate constant were measured in phosphate buffered saline (pH 7.4 and ionic strength $137 \mathrm{mM}$ ). 

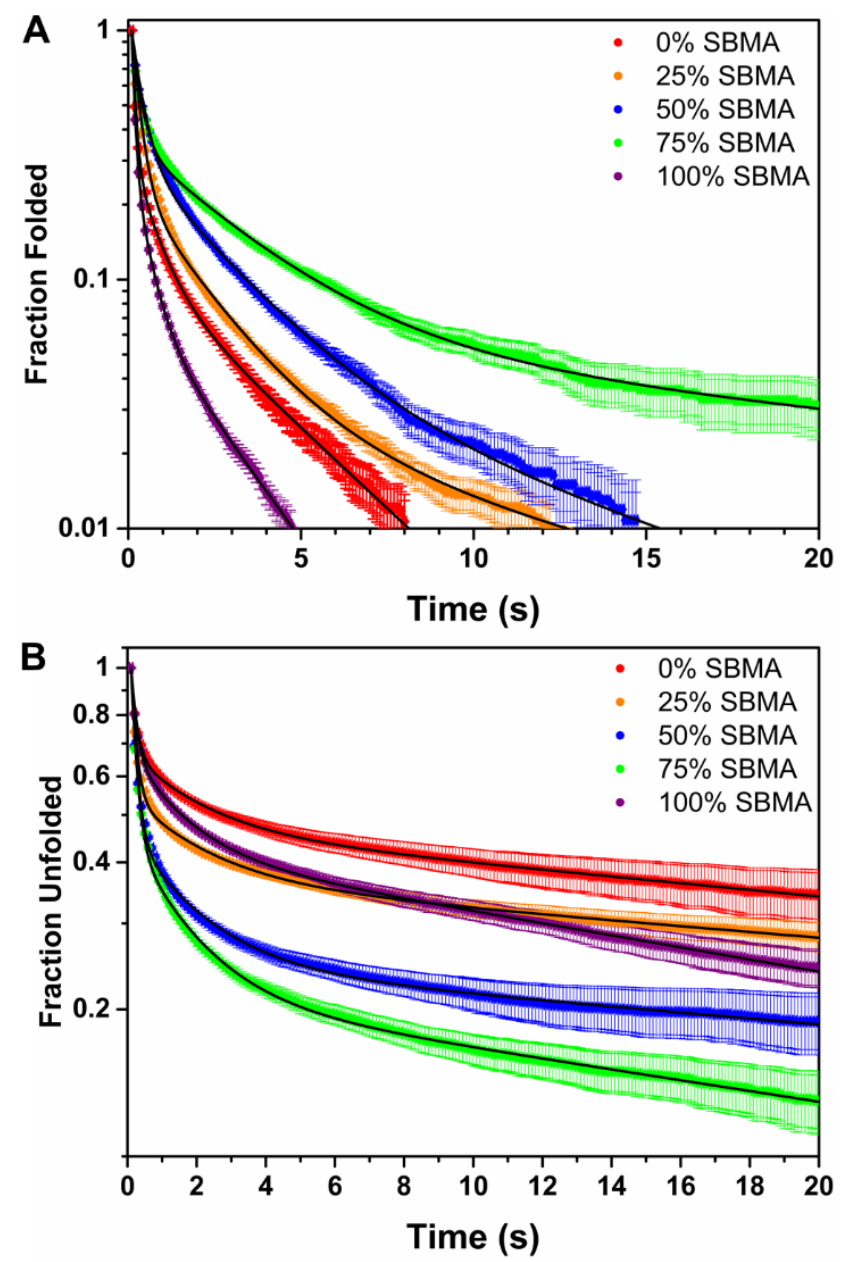

Figure S4. Complementary cumulative dwell time distributions for folded (A) and unfolded (B) FN on $100 \%$ OEGMA (red), 25\% SBMA/75\% OEGMA (orange), 50\% SBMA/50\% OEGMA (blue), 75\% SBMA/25\% OEGMA (green), and 100\% SBMA (purple) brushes. Each distribution was fit to an exponential mixture model (black line), which assumed a superposition of three firstorder apparent high-to-low FRET or low-to-high FRET transitions. Analysis of the model fit yielded the relative fraction and characteristic time constants for each sub-population in each distribution. Error bars represent a $68 \%$ confidence interval based on a Poisson distribution for each data point. Each distribution was generated from $\sim 10^{4}$ molecular trajectories. 

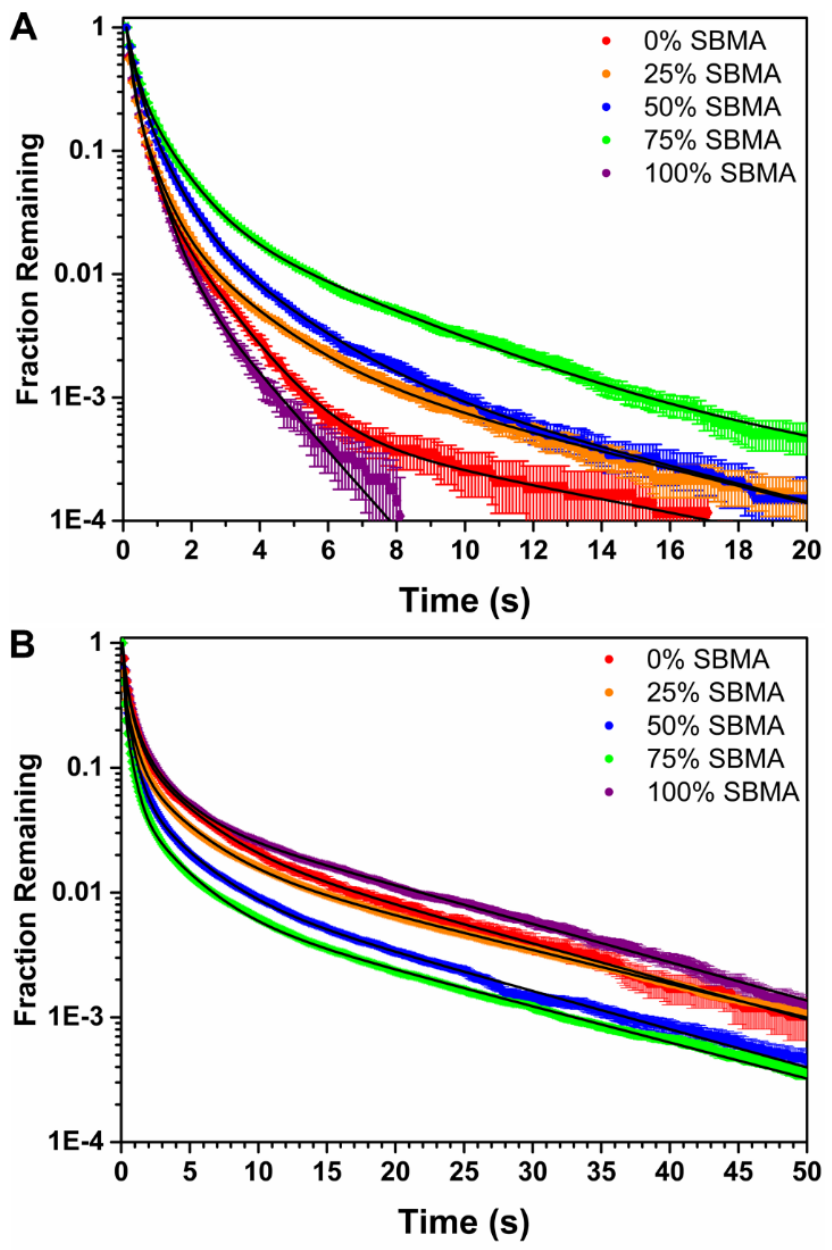

Figure S5. Complementary cumulative surface residence time distributions for $\mathrm{FN}$ on $100 \%$ OEGMA (red), 25\% SBMA/75\% OEGMA (orange), 50\% SBMA/50\% OEGMA (blue), 75\% SBMA/25\% OEGMA (green), and 100\% SBMA (purple) brushes as a function of folding state. Distrubtions for always folded and always unfolded FN are represented in panels A) and B), respectively. The distributions were fit to an exponential mixture model (black line), which assumed a superposition of four first-order desorption processes. Based on the fitting parameters, the characteristic surface residence time for each sub-population of folded or unfolded molecules on each surface was determined. Error bars represent a $68 \%$ confidence interval based on a Poisson distribution for each data point. Each distribution was generated from $\sim 10^{5}$ molecular trajectories. 


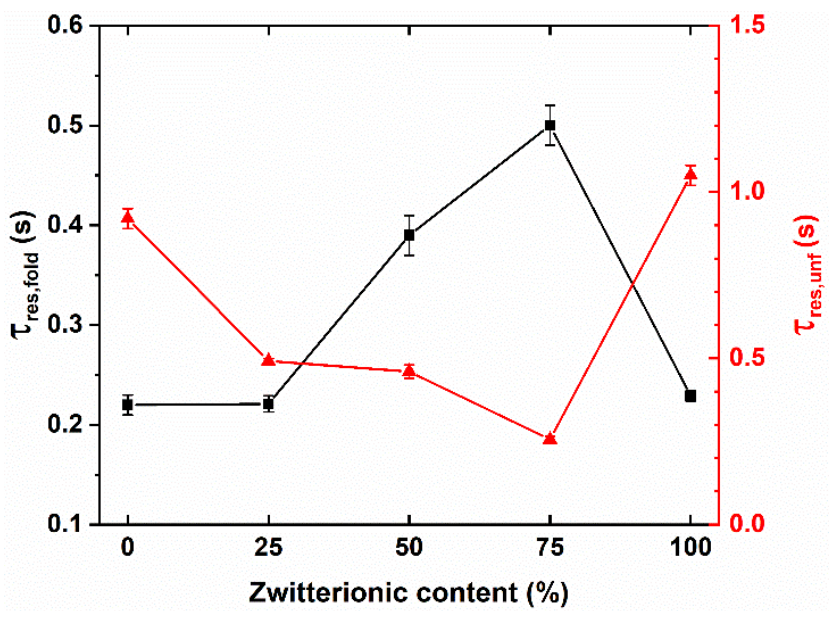

Figure S6. The impact of brush composition on the mean always-folded and always-unfolded state surface residence times, which were used to determine the apparent rate constants for desorption. Error bars represent the standard error associated with fitting complementary cumulative dwell time distributions.
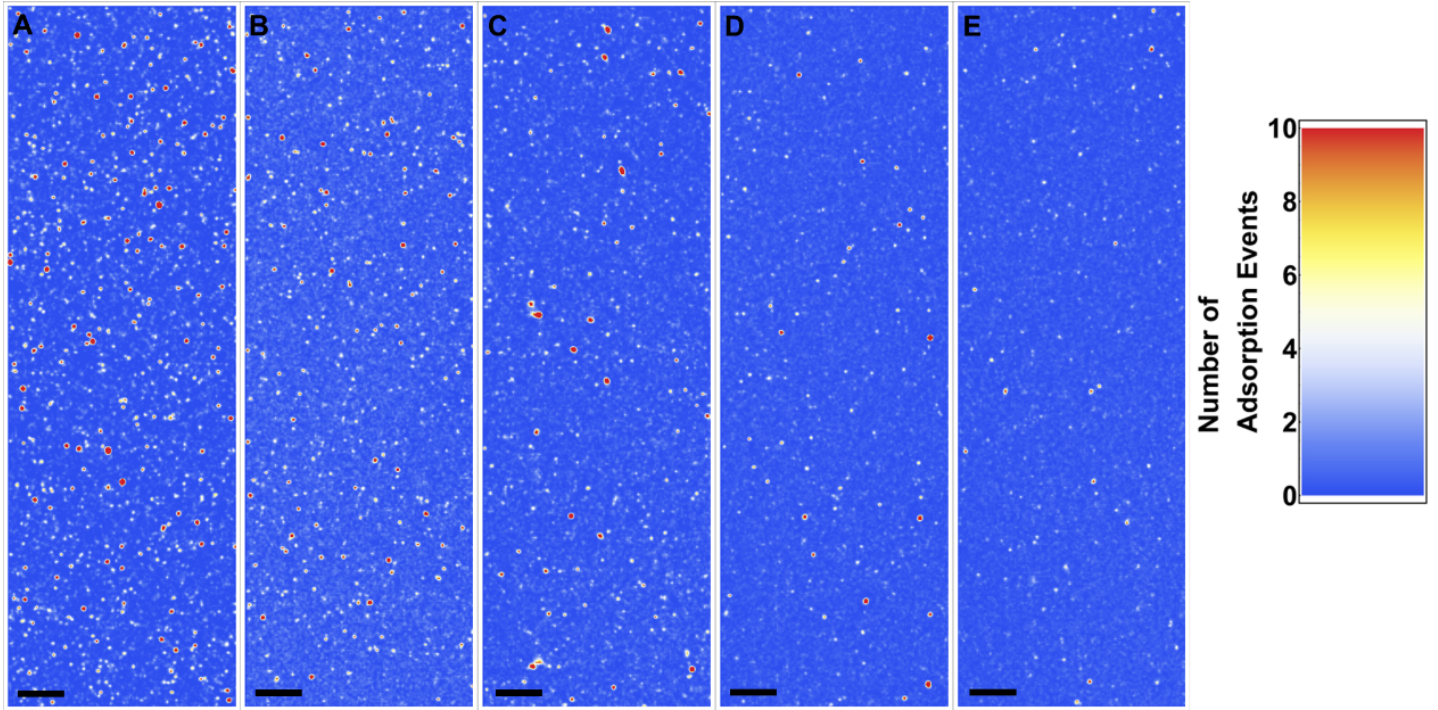

Figure S7. Super-resolution mapping of surface adsorption of NBD-X using mbPAINT, obtained from the accumulation of NBD-X trajectories on the polymer brushes for different chemical compositions: 100\% OEGMA (A), 25\% SBMA/75\% OEGMA (B), 50\% SBMA/50\% OEGMA (C), 75\% SBMA $/ 25 \%$ OEGMA (D), and 100\% SBMA (E). The numbers of adsorption events per pixel was counted and displayed in the form of a heat map. It can be observed that the number of strong adsorption sites systematically decreased as the content of SBMA increased on the brush surface. Scale bar $=5 \mu \mathrm{m}$. 


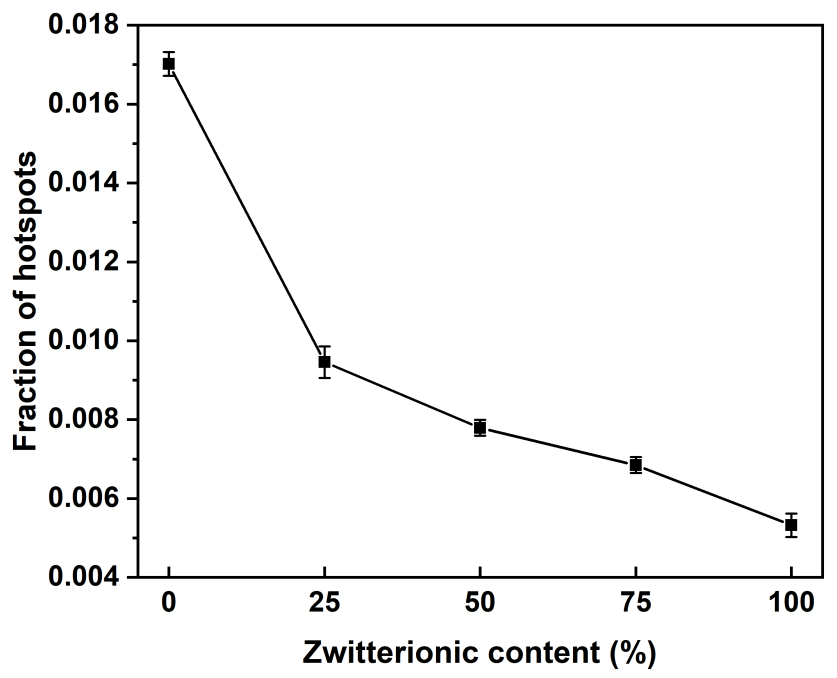

Figure S8. Quantitative analysis from mbPAINT maps using NBD-X as a probe molecule. Fraction of NBD-X trajectories that corresponded to hotspots (i.e., anomalously hydrophobic niches in the context of this system) with respect to the total number of trajectories for the different polymer brush compositions. A hotspot was defined as a site with at least five adsorption events. Error bars represent the standard error associated with randomly selecting different subsets of data (i.e., trajectories). 


\section{$\underline{\text { References }}$}

(1) Faulón Marruecos, D.; Kastantin, M.; Schwartz, D. K.; Kaar, J. L. Dense Poly(Ethylene Glycol) Brushes Reduce Adsorption and Stabilize the Unfolded Conformation of Fibronectin. Biomacromolecules 2016, 17, 1017-1025.

(2) Kastantin, M.; Faulón Marruecos, D.; Grover, N.; Yu McLoughlin, S.; Schwartz, D. K.; Kaar, J. L. Connecting Protein Conformation and Dynamics with Ligand-Receptor Binding Using Three-Color Förster Resonance Energy Transfer Tracking. J. Am. Chem. Soc. 2017, 139, 9937-9948.

(3) Hammill, J. T.; Miyake-Stoner, S.; Hazen, J. L.; Jackson, J. C.; Mehl, R. A. Preparation of Site-Specifically Labeled Fluorinated Proteins for 19f-Nmr Structural Characterization. Nat. Protoc. 2007, 2, 2601-2607.

(4) Peeler, J. C.; Mehl, R. A. Site-Specific Incorporation of Unnatural Amino Acids as Probes for Protein Conformational Changes. Methods Mol. Biol. 2012, 794, 125-134.

(5) Brandow, S. L.; Chen, M.; Dulcey, C. S.; Dressick, W. J. Formation of Aromatic Siloxane Self-Assembled Monolayers. Langmuir 2008, 24, 3888-3896.

(6) Kastantin, M.; Keller, T. F.; Jandt, K. D.; Schwartz, D. K. Single-Molecule Tracking of Fibrinogen Dynamics on Nanostructured Poly(Ethylene) Films. Adv. Funct. Mater. 2012, 22, 2617-2623.

(7) Walder, R.; Kastantin, M.; Schwartz, D. K. High Throughput Single Molecule Tracking for Analysis of Rare Populations and Events. Analyst 2012, 137, 2987-2996.

(8) McLoughlin, S. Y.; Kastantin, M.; Schwartz, D. K.; Kaar, J. L. Single-Molecule Resolution of Protein Structure and Interfacial Dynamics on Biomaterial Surfaces. Proc. Natl. Acad. Sci. 2013, 110, 19396-19401.

(9) Kastantin, M.; Schwartz, D. K. Connecting Rare DNA Conformations Molecule Resonance Energy Transfer. ACS Nano 2011, 12, 9861-9869.

(10) Kastantin, M.; Schwartz, D. K. Identifying Multiple Populations from Single-Molecule Lifetime Distributions. ChemPhysChem 2013, 14, 374-380.

(11) Weltz, J. S.; Schwartz, D. K.; Kaar, J. L. Surface-Mediated Protein Unfolding as a Search Process for Denaturing Sites. ACS Nano 2016, 10, 730-738.

(12) Walder, R.; Nelson, N.; Schwartz, D. K. Super-Resolution Surface Mapping Using the Trajectories of Molecular Probes. Nat. Commun. 2011, 2, 1-7.

(13) Chen, J.; Bremauntz, A.; Kisley, L.; Shuang, B.; Landes, C. F. Super-Resolution MbPAINT for Optical Localization of Single-Stranded DNA. ACS Appl. Mater. Interfaces 2013, 5, 9338-9343. 